JOHN KNODEL

\title{
FOCUS GROUPS AS A QUALITATIVE METHOD FOR CROSS- CULTURAL RESEARCH IN SOCIAL GERONTOLOGY
}

\begin{abstract}
The focus group approach for collecting qualitative data can be usefully applied in social gerontological studies, both in a single setting and cross-culturally. The experiences of the research teams participating in the Comparative Study of Asian Elderly in employing focus groups are described, and their advantages and disadvantages as a general method for gathering basic qualitative data are discussed. While the method has promise, it also should be recognized that conducting focus group research within the context of a comparative study compounds the considerable time, effort, and funds that focus group research for basic social science already requires.
\end{abstract}

Key Words: focus groups, qualitative methodology, social gerontology research, cross-cultural research

\section{INTRODUCTION}

A variety of techniques for collecting qualitative data is available to social researchers who study aging, the elderly, intergenerational exchanges, and related subject matter, whether doing so in a single social setting or conducting crossnational or other types of comparative studies. These techniques include participant observation (often involving lengthy residence in a community), in-depth interviews (with key informants or representatives of some targeted subset of the population), case studies, and analysis of written textual material such as novels, letters, and diaries. Moreover, secondary sources of qualitative information such as ethnographies can be drawn upon. Focus group discussions represent yet an additional technique.

The present article describes focus group methodology and its potential as a research technique in the area of cross-cultural gerontology drawing on experience using the method in conjunction with the broader Comparative Study of the Elderly in Asia described in the introduction to this issue of the Journal of Cross-Cultural Gerontology. The focus group component involved a collaboratively designed and coordinated data collection and analysis effort carried out by affiliated local researchers in the Philippines, Singapore, Taiwan, and Thailand. As such, it represents the first cross-cultural social science study based on focus group methodology.

The present article starts with a description of the focus group approach in general, mentioning some of its advantages and disadvantages as a method for basic qualitative social research. This introduction is followed by a description of our experience with focus groups in the context of our comparative research project on Asian elderly. Special emphasis is given to issues involving the appli- 
cation of the method for cross-cultural research in social gerontology. Substantive results are reserved for the five following articles: four presenting country specific results and the one dealing with comparative results. Only methodological issues are addressed here.

\section{THE FOCUS GROUP APPROACH}

The basic idea of the focus group method is to generate a discussion on preselected topics of interest to the researcher among a small group of individuals from a target population defined in terms of characteristics relevant to the research topic. The group discussion 'focuses' on a relatively narrow set of topics thereby giving the method its name. A moderator following prepared guidelines introduces the concepts to be discussed, asks open-ended questions to get the discussion underway, encourages participants to talk and interact with each other, and guides the discussion to keep it on track. A note-taker is also present but normally does not take part in the discussion. The group typically involves 5 to 10 participants from the target population. The discussion is usually tape recorded and transcribed with the transcripts serving as the data for analysis. ${ }^{1}$

As the above definition implies, there is a rather specific methodology involved with focus group research. It does not encompass just any group discussions but those following particular procedures. Moreover, focus groups are intended to be more than just a group interview in a simple question-answer format. Critical to the technique is that participants engage in some level of discussion and not simply reply to the moderator in response to direct questions.

While there are many legitimate purposes for which focus groups can be used, our own application of the method and the discussion that follows is oriented towards basic social research. This application is by no means their most common use. Despite origins in sociology at least half a century ago, focus group discussions became best known subsequently as a method of marketing research, used for quick assessment of consumers' impressions and feelings towards specific products or advertising concepts. ${ }^{2}$ They also have been used with some frequency as a tool in evaluative and applied research projects (Krueger 1988). More recently, the technique is again begin used by social scientists to conduct basic research. As a result the method continues to be refined and adapted to increase its suitability for this purpose, and a growing literature on the use of focus group methodology from a social research perspective is emerging. ${ }^{3}$

\section{Some Advantages}

Among the key advantages of focus groups when they function properly is that they generate discussion among the participants. Comments by one participant can stimulate others' thoughts about the topic and lead to verbal reactions by them. This group interaction process is missing in data collection techniques that involve private interviews, be they of a structured questionnaire type or an open- 
ended in-depth interview. Initial comments or revelations by the bolder, more out-spoken participants on a potentially sensitive issue can ease the way for the remaining participants to speak frankly.

Compared to typical ethnographic or other observational type approaches to qualitative research, the focus group method involves relatively limited contact with the target populations, not requiring extended residence with any particular group. Moreover, the imposition of a fairly structured set of guidelines for moderators to follow when directing the discussions and their systematic implementation across groups, results in relatively structured data. From a traditional ethnographic point of view these features may be seen as drawbacks. For others, however, they serve to make collection of qualitative data more practical and to considerably facilitate comparative analysis of the data when more than one cultural setting is involved. The quasi-structured nature of the data is also likely to add to the appeal of focus groups for the large body of social researchers who have been trained in the sample survey tradition where structure and systematic data collection procedures are paramount.

To be sure, in-depth community studies by ethnographers and anthropologists can root attitudes and behavior far more solidly in their social and cultural context than can focus group research alone. However, any single ethnographic study is typically based on only one community. Moreover, ethnographic overviews of findings from the broader body of studies rarely make clear just where the supporting evidence for particular generalizations originate. The more systematic nature of the data generated through focus groups and their suitability for being conducted in a number of communities in the same study permits more confidence for the analyst when differentiating what is a local peculiarity from more general patterns in the society at large. This is particularly important for cross-national comparative studies which are chiefly interested in contrasting relatively general features of the societies under consideration.

Another advantage of the focus group method is that the resulting transcripts more easily permit re-analysis or further analysis by other researchers than would most ethnographers' field notes. Since the transcripts are normally word processed anyway, they can be archived in a reasonably accessible form or even issued in full in hard copy for use by others with relatively minor effort. Indeed, the Taiwan research team in the present project has actually issued the full set of transcripts (in Chinese) in the form of a self-standing publication.

\section{Some Limitations and Liabilities}

The typically small number of total participants and their purposive selection renders focus group data inappropriate for quantitative analysis beyond merely distinguishing more commonly voiced views from those that are seldom mentioned. Results are not representative in any statistical sense although the views expressed can still be more or less typical for the particular target group. As with all qualitative methods, considerable subjective judgement is required in the process of data collection and interpretation of results thus heightening the risk 
of introducing personal biases into the study. Moreover, the fact that the quality, and perhaps even the validity, of data generated from focus group sessions depends to some extent on the personality and skills of the moderators is somewhat unsettling (Khan, Ankar, Patel, Barge, Sadhwani, and Kohle 1991; Khan and Manderson 1992). This can be an even greater liability in a comparative study where the ease with which the focus groups can be conducted may vary across cultures and where the qualifications of the research teams may also vary.

Less frequently recognized is the fact that the amount of effort (and expense) in properly carrying out a focus group study for social science purposes is formidable. The considerable time and effort required for sound data collection, thorough data processing, and careful and systematic analysis of transcripts are often unanticipated and inadequately planned for in time and financial budgets, especially by those attempting focus group research for the first time. As a result, the quality of the data and findings often suffer. The prevalent, but mistaken, impression that the method is quick and simple to implement is undoubtedly engendered by its rather different use in marketing and evaluation research.

While focus groups can be good at eliciting perceptions, opinions, and attitudes from participants, they are a poor medium for obtaining detailed or systematic behavioral data of a personal nature. Accounts of individual experiences inevitably arise during a focus group session, and general observations of behavior in the community can be solicited, but it is usually inappropriate to probe the details of a particular individual's situation and the history that led up to it in the course of a group discussion. For example, eliciting detailed and coherent accounts of the life course events that led to particular support and care arrangements in old age would be awkward within a focus group context. In-depth interviews conducted on a one-to-one basis would seem considerably better suited for this purpose. ${ }^{4}$

The appropriateness of focus groups is also limited for soliciting the views of persons whose circumstances deviate substantially from the modal situation with respect to the research topic if other participants in a focus group session conform more closely to the norm. For example, in societies where elderly are typically supported by their adult children, those not receiving such support may have little to say about these matters in a group composed primarily of participants who receive it. ${ }^{5}$ Nevertheless, the views of such persons can be of particular interest to the social researcher precisely because of their marginal position in the society. Unless a whole group of individuals with similar non-modal circumstances can be formed, unlikely in most field sites given their limited occurrence, it is probably more appropriate to use in-depth interviews to gather equivalent qualitative data from them.

In brief, focus groups are not intended to be a substitute for quantitative research such as exemplified by sample surveys nor as an exclusive technique for qualitative data collection. As with any social research method, focus groups have strengths and limitations that make them more suitable for certain purposes and less suitable or even totally inappropriate for others. In many contexts, how- 
ever, they can be a useful method whether used on their own or in conjunction with other quantitative or qualitative approaches.

\section{CROSS-CULTURAL RESEARCH}

Since there have been no prior cross-cultural focus group projects within the framework of basic social research, there is no literature addressing issues specific to such endeavors. The following commentary derives from the experiences of our own project and it is only impressionistic. Indeed very little systematic research on focus groups methodology has been conducted, especially within the context of social science (Morgan 1993b). Instead, recommended procedures and rules are generally derived intuitively and supported at best by anecdotal evidence only.

The clearest lesson from our experience is that conducting focus group research within the context of a comparative study adds substantially to the already considerable time, effort, and funds that focus group research for basic social science already requires. This is particularly so when a common standard of relatively good quality data collection and analysis is to be maintained. Periodic project-wide workshops were essential and extremely useful, but they required considerable time, effort, and funds to organize and attend. In addition, the need to proceed in a stepwise fashion with each step being completed by all four country teams before the next step began considerably lengthened the time needed to conduct our research and analysis compared to the time that would have been required had each country independently conducted its study. However, this sequential procedure is probably inevitable in a cross-national project if any adequate degree of coordination is desired.

\section{Coordination and Standardization}

Only one of the four country teams in our project involved members who had had any substantial prior experience conducting focus group research. Most members were trained as social demographers and had a quantitative research orientation (the Singapore team with a social work background was the exception). In part to compensate for this lack of experience, a practical manual on conducting focus group research was prepared with the specific subject matter of the project in mind (Knodel, Sittitrai, and Brown 1990). This helped standardize the procedures that were followed.

The focus group research teams met together in a total of 7 international workshops during the course of the project. These included two special workshops that were held exclusively for the subset of project members responsible for the focus groups and the five annual overall project workshops, during which several days were devoted specifically to matters conceming the focus group component. The workshops served as the major mechanism for facilitating and coordinating the research efforts. 
The themes specific to the focus group component that were covered at the overall project workshops involved, more or less in sequence: a) determining in detail the specific topics to be addressed by the focus group component including development of country-specific discussion guidelines; b) development of study designs; and c) training (by project members with focus group research experience) in field work procedures and techniques of analysis. The two special workshops were held after data collection was completed and were devoted to analysis of the data. The workshops also served the function of providing meaningful deadlines for pressuring the various teams to complete various steps of the research process.

Even with all the workshops we held, the effort to achieve reasonably common procedures in field work and analysis was only partially successful. In part, this was because some country team members were unprepared for how demanding the data collection, data processing, and, above all, analysis, would be of their own time. Tasks that might be appropriate to assign to assistants when conducting quantitative research often require a more extensive role of the principle investigator in qualitative research. Particularly in the analysis stage, relatively little can be delegated to assistants. In the end there is no substitute for the analyst reading (and re-reading) the transcripts multiple times if the contents are to be properly digested. In focus group analysis, there is no equivalent to the situation in survey analysis where much of the tabulation can be relegated to a skilled programmer.

In addition to practical constraints on researchers' time and efforts, differences of research style also came into play, possibly affecting the nature of the qualitative data collected and their interpretation when analyzed. Moreover, in any cross-cultural project there is tension between the need to adapt the study design, research instruments, and field work procedures to suit the particular circumstances of each country setting and the need to maximize comparability across settings. Striking the appropriate balance is never easy. It is all the more difficult when the research approach is a new one, and hence unfamiliar, to most participating investigators. Face-to-face meetings at the workshops where the relevant matters can be concretely discussed helped this process considerably. Compromises and judgement calls are necessarily involved. Not all differences are easily resolved, however, especially within the limited time of a workshop.

\section{Issues Related to Study Design}

To appropriately judge the lessons that our experience provides, it is essential to understand the general design of the focus group component of the overall project. The number of focus group sessions held varied by country, ranging from 16 to 26. Most commonly, each session involved 7-9 participants. The discussions dealt with a range of issues centering around expectations for support in old age, intergenerational exchanges, and the position of the elderly in society. Living arrangements, the topic addressed by the following substantive articles in this special issue, was only one of a number of related topics covered. 
In each of the countries, separate sessions were conducted with elderly people (defined as aged 60 and over) and adult children (typically of elderly parents in their $30 \mathrm{~s}$ and $40 \mathrm{~s}$ ). In all countries, most groups were segregated by better and worse socio-economic status (or education). In all but Singapore, both rural and urban groups were formed. In Singapore and Taiwan, groups were also segregated by ethnicity. In addition, all sessions in the Philippines and most in Taiwan were separated by gender.

The purpose of defining groups in terms of key characteristics was twofold. A common intuitive principle in focus group research is that participants feel more comfortable and hence more willing to talk openly when they are among social peers rather than when mixed together with persons of notably different social positions. Thus one purpose was to facilitate frank group discussion. In the cases of the Philippines and Taiwan, the local researchers believed that sessions would flow more freely in single-gender groups. Otherwise, they suspected the discussion would be dominated by male participants. Our impression in all countries is that grouping persons with similar characteristics together (rather than mixing them in the same session) did indeed serve to facilitate discussion, although we did not experiment with alternative mixes of characteristics other than gender in the case of Taiwan. In fact, in this particular case, the Taiwanese investigators had the impression that mixing men and women did not inhibit discussion in those groups as they had expected it would. This finding is verified by the fact that counts of the number of times participants spoke in the mixed gender Taiwanese groups show that women spoke on average as many times as men. The same was true for the Thai groups, all of which were mixed?

Some of the characteristics incorporated into the design to define subsets of groups were also expected to be of substantive or theoretical interest. Thus the second purpose of holding separate sessions with differently specified target populations was to permit comparisons along several dimensions (e.g., intergenerational, urban-rural residence, ethnicity, and socio-economic status). In practice, the ability to make such comparisons in the analysis varied among the countries. Only where rather stark differences were associated with the characteristic (or combination of characteristics) could this aspect of the design serve the intended purpose. Elsewhere, it proved too difficult to discern clear patterns in the analysis along these lines. In part, this problem arose because each group had idiosyncratic aspects (related to features such as moderator style and the mix of personalities of the participants) that are unrelated to their position in the design scheme but which influence the flow and nature of the discussion.

The clearest case where intra-country contrasts could be successfully made based on the design was Singapore, where the cultural differences between the three main ethnic groups are pronounced and hence associated with considerable differences in attitudes and practices regarding support and care of the elderly. Even here, however, the utility of the division by ethnicity for making comparisons was tempered by the small number of sessions among Indians and Malays. In Taiwan, the investigators felt that the ethnic distinction between Mainlanders 
and others (Fukienese and Hakkanese) revealed clear patterns of differences on some issues under study. In Thailand, contrasts between the rural and Bangkok groups, especially middle class Bangkok groups, are at least suggestive of a patterned difference, but other clear and convincing contrasts were not obvious. Likewise in the Philippines, patterns of differences along the design dimensions were not obvious.

Despite the lack of more obvious intra-country differences emerging, incorporating these various dimensions into the design still proved useful for the analysis by helping establish the generality of particular views within the entire society rather than differences between subgroups. To the extent that similar opinions and experiences were expressed by differently defined subsets despite the idiosyncrasies involved in the conducting of the different sessions, we can be relatively confident that the views being tapped reflect shared cultural values and practices within the broader populations. Indeed, emphasizing what is common among the different groups rather than what is different is probably a sounder use of the design in most situations, given that only a small number of groups are typically available for each subset.

Most significant from the perspective of cross-cultural studies, where the countries themselves form the most important basis for comparisons, major differences (as well as similarities) are quite evident among the countries. Assessing these differences is potentially complicated by the fact that country findings are necessarily associated with different research teams whose varying styles may affect on the results despite our attempts to minimize such effects by following more or less similar field work and analytical procedures. Nevertheless, cultural differences between countries are usually pronounced enough for focus groups to reveal some fairly obvious contrasts. Moreover, the plausibility of the focus group results can be judged by drawing on related information from other sources such as surveys and ethnographies.

Our intention to draw contrasts in views along a number of the dimensions used to define groups drove up the number of sessions planned in each country. As indicated, however, we are finding it difficult to make many intra-country contrasts with confidence. Instead, most of the views that we document are rather robust in the sense that they emerge repeatedly in group after group. Thus, with the exception of Singapore where sharp cultural differences exist among the three main ethnic groups, the number of focus groups we conducted was probably more than sufficient to determine the general views of the populace towards the issues addressed. The findings of our analyses in the other three countries would probably differ little if the number of groups had been substantially fewer. Nonetheless, the larger number of groups does help increase our confidence in the findings even if they do not alter the results significantly.

\section{Issues Related to Data Processing and Analysis}

Processing and analyzing focus group transcripts involve quite different procedures from those involved in the processing and analysis of survey or other 
types of quantitative data. Our particular approach in the Comparative Study of the Elderly in Asia involved several major features that all teams were asked to follow: a) every focus group session was to be fully transcribed; b) the word processed transcripts were to be code-mapped; c) The Ethnograph software package was to be used for retrieving coded segments during analysis; d) overview grids were to be composed to enable statements to be made about the generality of particular views; e) two or more researchers were to read each transcript and compare impressions of its content to increase reliability of the analysis; $f$ ) other sources of information bearing on the topic were to be drawn on to help assess the plausibility of results and place them in perspective. Not all teams actually followed these recommended procedures fully but at least they attempted to in some degree. Each of these steps is commented on below.

\section{Transcription (and translation)}

An average focus group session lasted one and a half to two hours. Transcribing the discussion in full is thus a major task. For some purposes, transcription is not necessary and a summary account compiled at the time of the session or by listening to the tapes might suffice. However, when focus groups are conducted for basic social research purposes such as in our project there really is no substitute for full transcription. Fortunately this aspect of data processing can be delegated to someone other than the principal investigators. The ideal person to do the transcription is usually the research assistant who served as note-taker, whose main function is to jot down enough information at the time of the actual session to be able to identify each speaker.

It is essential, however, for the principal investigator to check the completeness of the transcriptions. It is unfortunately common for inexperienced transcribers to leave out parts of the discussion (especially side comments or conversations or repeated statements). In the case of the Thai team, significant omissions in the transcriptions were discovered only after a substantial number of transcriptions had been done. Correcting them caused considerable delay in the data processing schedule.

In comparative research projects such as ours where international teams of researchers are involved or even at the national level when different dialects or languages exist in a country, it is often necessary to translate the transcripts into a common language. While the ideal situation is to have two sets of transcripts, one in the original language and the other in a common language, this did not always prove practical within the constraints of budget and time in our project. Indeed, the necessity for translating and the manner in which it was done varied with the particular country team. When regional dialects were involved, statements were usually translated directly into a common language in the course of transcription.

\section{Code-mapping}

Central to an analysis of qualitative data such as focus group transcripts is the process of coding the text into analytically distinct segments which can then be 
examined together when drawing conclusions concerning one or more of the topics and related concepts under investigation. In addition, coding can identify segments for some practical purpose such as use as quotations. In practice, the analyst reads the transcript and marks it with appropriate codes in the margins where segments corresponding to different topics and concepts of interest start and end, thereby effectively mapping the transcript. ${ }^{8}$

It is unrealistic and unnecessary to code a transcript in great detail the first time through. Many concepts and subjects that will be useful to code will only become apparent as the transcripts are re-read in the course of analysis and the actual writing up of the results. Moreover, probably the most important use of code-mapping will be to retrieve segments that correspond to topics and subtopics explicitly incorporated in the discussion guidelines used by the moderator in the course of the focus group sessions (Knodel 1993). That way, when writing up results referring to a particular topic or sub-topic, the relevant segments can be quickly located and reviewed.

\section{Use of the Ethnograph}

Retrieval of coded segments is greatly facilitated by the use of computers and software such as The Ethnograph, which allows the user to enter the codes and indicate the location of associated text segments onto a computerized file of text. ${ }^{9}$ For example, if segments related to the discussion of the desired gender of the child with whom elderly normally coreside had been assigned a specific code, all segments related to this in the transcripts of all or any subset of focus group sessions could be quickly and conveniently retrieved for the analyst to read and digest when determining findings on this topic.

All the research teams in our project used The Ethnograph program but to differing degrees. In retrospect, insufficient time was devoted to training during the workshops on how to best take advantage of the program's features. In some cases team members coded in too great detail and ignored the more useful straightforward coding of the topics and subtopics as explicitly laid out in the discussion guidelines. As with any new software package, and especially one that is being used for an unfamiliar style of analysis, utility increases as one becomes more familiar with its features.

\section{Overview Grids}

Given the nature of focus group data, considerable subjective judgement is required when examining and interpreting the content of the transcripts. Often several alternative views are expressed within the same group and even, at times, by the same person. Moreover, although views can be fairly uniform across groups for some issues, for others quite different views may emerge or the issue may not arise at all. It thus helps to have some systematic way to summarize the content of the discussions by topic and group to aid interpretation and to help minimize personal bias in drawing conclusions. For this purpose, we followed 
the practice of constructing overview grids (Knodel 1993). Such a grid would typically have topic headings or particular views on one axis and focus group session identifiers on the other. The cells contain brief summaries of the discussion for each group concerning each topic as well as other information such as indications of the extent of elaboration or strength of opinion.

The grid provides a basis for determining, in a relatively systematic way, how common particular views are as well as revealing if patterns of differences are evident along lines that demarcate the target populations included in the study design. The use of the overview grid for these purposes, however, should not be mistaken for a type of mechanical quantitative analysis. Rarely is there any point in reporting actual counts of the times a view is expressed or the number of groups in which it arises. Instead, only broad distinctions would be stated, differentiating views which are common from those that arise only rarely. The overview grid is a tool for helping the researcher make the analysis more accountable to the data (i.e., the transcripts). Constructing a grid is just one part of the analysis process, most of which consists of reading and re-reading the appropriate segments of the transcripts dealing with each sub-topic being addressed.

While it is not imperative to have code mapped the transcript prior to constructing an overview grid, having done so facilitates the task. Moreover, use of The Ethnograph program can also help in the process. Each segment of each transcript relating to each sub-topic in the grid can be called in turn to the computer screen (or printed out in hard copy). The analyst can then read them as they appear and summarize the content in the appropriate cells of the grid.

\section{Reliability Checks on Interpretation}

Utilizing a team approach involving several researchers when analyzing focus group data can contribute to the reliability of the interpretation. The process consists of team members independently reading the transcripts and then comparing impressions with each other. Disagreements can then be discussed and generally resolved by reviewing the transcripts together and tracking down the source of the disagreement. By having each team member independently construct an overview grid, it is possible to establish considerable reliability early in the interpretive process. If both original language transcripts and translations are being used, a team approach is essential so that at least one member analyzes the original language version. To varying degrees, the country teams all followed this procedure.

The accuracy of the interpretive analysis is also enhanced if those who eventually analyze the data are intimately involved with the actual data collection (i.e., present at the focus group sessions and possibly even serving as moderator). This dual role eliminates considerably the 'distance' between the analyst and subjects being studied that is so often found in quantitative social science research, i.e., studies in which only interviewers and not the eventual analysts have contact with respondents. For all four country teams in our project, this dual role strategy has been followed to a large degree. 


\section{Drawing on External Evidence}

Findings from focus group research need to be judged in light of what we already know about the topic from other sources. Considerable subjective judgment is involved when interpreting focus group discussions. Not all statements can be taken at face value but rather require interpretation. Sometimes it is necessary to 'read between the lines' in order to make sense of contradictions in the discussions. While knowing the context in which comments are made helps, information, of both a quantitative and qualitative nature, available to the researchers from external sources can also be useful. Determining the degree of convergence between focus group data and other sources is important for assessing the plausibility of the focus group results. To the extent that the various sources of evidence converge, the results can be presented with justifiably greater confidence. Even when there is not close convergence, the contrasts can be instructive and serve as a useful basis of reflection on the validity of both the focus group data and those from external sources.

The subsequent analyses of living arrangements illustrates how qualitative data generated through focus groups can be useful in the pursuit of cross-cultural gerontology. By selectively referring to available survey evidence, the following articles should also make clear that the value of the focus group method is considerably enhanced when other types of information are available for comparison. As social researchers increasingly recognize the advantages of having a combination of qualitative and quantitative data in their search for understanding of the phenomena they study, focus group methodology is likely to gain greater prominence. Hopefully, this special issue of the Journal will serve to illustrate the utility of the method for cross-cultural gerontology.

\section{ACKNOWLEDGEMENTS}

This article benefited from extensive discussion of many of the issues addressed during a workshop held in Bangkok, Thailand in December 1993 attended by Lita Domingo, Mei-lin Lee, Harvey Lin, Kalyanti Mehta, Maliki Bin Osman, and Chanpen Saengtienchai.

\section{NOTES}

1. In cases where a 'quick' study is considered adequate, transcription and even tape recording may be skipped and the detailed notes of a note-taker can serve as the basis for the analysis. Such short-cuts, however, are rarely suitable for serious social research purposes.

2. The earliest description of a technique resembling focus groups appears to be Bogardus (1926). More commonly cited is the work of Merton and associates in the 1940s (e.g., Merton and Kendall, 1946) and particularly the book that emerged from their work (Merton, Fiske, and Kendall 1946). A good example describing focus group research from a marketer's point of view is provided by Greenbaum (1988).

3. A particularly up-to-date account is provided by the volume edited by David Morgan (1993a). See also Knodel, Pramualratana, and Havanon (1988); Morgan (1988); Khan et al. (1991); and Khan and Manderson (1992). 
4. An excellent example of the usefulness of a case study approach (in connection with a mini-survey) for providing insights into living arrangements and other aspects of the elderly and their care givers is provided by the work of Caffrey (1992a and 1992b) published in this journal. An example of a more full-scale ethnographic study of the elderly in Thailand illustrating the richness of data and insights from this approach is provided by Pramualratana (1990).

5. This appears to be what happened in one Thai focus group where an elderly male participant who lived with only his wife appeared quite talkative when we recruited him but said little in the focus group session made up of other participants who were living with or next to children.

6. The focus group research component of the Comparative Study of the Elderly in Asia began at the start of the overall project in early 1989 with country teams being formed and initially drafting their plans in preparation for the first project-wide workshop held mid-year. At that workshop all 4 country teams met together (along with project associates not directly assigned to the focus group component) to coordinate study designs and topics to be covered in the discussion guidelines. This was followed by pretesting in each country. The actual fieldwork took place during 1990-1992, the particular timing depending on the country. Since fieldwork need not be continuous, it was typically spread over periods from half a year to a year and a half. The time required to organize and hold sessions also varied. A rough idea is provided by the Thai case. Carrying out a set of 4 complementary sessions ( 2 with elderly and 2 with adult children stratified by better and worse socio-economic status) by a team of 5 or 6 researchers and assitants took about a week in each of the 4 regions. Total accumulated time spent by the team in actual fieldwork, including the Bangkok groups, took about 2 and a half months.

Data processing (transcription, translation, word processing) was also spread over fairly long periods of time. Since it can be done following each session it need not wait for all fieldwork to be completed before starting. In the Thai case, initial transcription in Thai of a 2 hour focus session typically took a research assistant about 12 to 15 hours of concentrated work. To this must be added the time to check and correct the transcription. In the Thai case translation added very substantially to the data processing because it was often done on consignment by persons who did it bit by bit. With some experience, an analyst could do a basic code-mapping (see below) of a transcript in about 4 hours of concentrated effort. Thus several weeks of real concentrated work are required just to code-map the complete set of 18 to 26 transcripts. It is difficult to estimate a time for the actual analysis as it will vary greatly by individual and the scope of the analysis planned. However, the fact that it took over 5 years from the start of the project to complete the set of articles for this special issue perhaps is the most telling fact indicating how time consuming and demanding comparative focus group research for basic social science purposes can be.

7. In the Thai case, the men spoke somewhat more frequently in the elderly groups while among the adult children groups the women spoke more frequently.

8. The term code-mapping is borrowed from the manual of The Ethnograph program (Seidel, Kjolseth and Seymour 1988).

9. The Ethnograph software is available through Qualis Research Associates, P.O. Box 2070, Amherst, MA 01002, USA.

\section{REFERENCES}

Bogardus, E.M. 1926 The Group Interview. Journal of Applied Sociology 10: 372-82.

Caffrey, R.A. 1992a Family Care of the Elderly in Northeast Thailand: Changing Patterns. Journal of Cross-Cultural Gerontology 7(2): 105-116.

Caffrey, R.A. 1992b Caregiving to the Elderly in Northeast Thailand: Changing Patterns. Journal of Cross-Cultural Gerontology 7(2): 117-134. 
Greenbaum, T.L. 1988 The Practical Handbook and Guide to Focus Group Research. Lexington, MA: Lexington.

Khan, M.E., M. Anker, B. Patel, S. Barge, H. Sadhwani, and R. Kohle 1991 The Use of Focus Groups in Social and Behavioral Research - Some Methodological Issues. World Health Statistics Quarterly 44: 145-148.

Khan, M.E. and L. Manderson 1992 Focus Groups in Tropical Diseases Research. Health Policy and Planning 7(1): 56-66.

Knodel, J. 1993 The Design and Analysis of Focus Group Studies in Social Science Research. In Successful Focus Groups: Advancing the State of the Art. David Morgan, ed. pp. 35-50. Newbury Park, Ca: Sage.

Knodel, J.A. Pramualratana, and N. Havanon 1988, Focus Group Research of Fertility Decline in Thailand: Methodology and Findings. In Micro Approaches to Demographic Research. J.C. Caldwell, A. Hill, and V. Hull, eds. Pp. 41-55. London: Kegan Paul International.

Knodel J.W. Sittitrai, and T. Brown 1990 Focus Group Discussions for Social Science Research: A Practical Guide with an Emphasis on the Topic of Ageing. Comparative Study of the Elderly in Asia, Research Report No. 90-3. Ann Arbor: Population Studies Center, University of Michigan.

Krueger, R.A. 1988. A Practical Guide for Applied Research. Newbury Park, Ca., Sage.

Merton, R.K. and P. Kendall 1946 The Focused Interview. American Journal of Sociology 51: 541-57.

Merton, R.K., M. Fiske, and P. Kendall 1956 The Focused Interview. Glencoe, I11.: The Free Press.

Morgan, D. 1988 Focus Groups as Qualitative Research. Newbury Park, Ca., Sage.

Morgan, D., ed. 1993a Successful Focus Groups: Advancing the State of the Art. Newbury Park, Ca, Sage.

Morgan, D. 1993b Future Directions for Focus Groups. In Successful Focus Groups: Advancing the State of the Art. D. Morgan, ed. Pp. 35-50. Newbury Park, Ca: Sage.

Pramualratana, A. 1990 Support Systems of the Old in a Rural Community in Thailand. Ph.D. Thesis. Australian National University, Canberra, Australia.

Seidel, J.V., R. Kjolseth, and E. Seymour 1988 The Ethnograph: A Users Guide. Littleton, Co.: Qualis Research Associates.

Population Studies Center

University of Michigan

1225 S. University

Ann Arbor, Michigan 48104-2590

U.S.A 